\title{
MULTIPROVA: APRIMORANDO A AVALIAÇÃO COM O USO DA TECNOLOGIA
}

\author{
André Bessa (abessa@ect.ufrn.br), ECT/UFRN \\ Diego Rodrigo Cabral Silva (diego@ect.ufrn.br), ECT/UFRN
}

\begin{abstract}
Resumo. Ações que avaliem e realimentem o processo de ensino-aprendizagem individual dos estudantes requerem uma estratégia eficiente de gestão do grande fluxo de informações que são geradas. Este artigo discute uma metodologia de avaliação formativa baseada nas funcionalidades do software Multiprova: geração de questões diferentes para cada estudante; uso de figuras, gráficos e fórmulas; correção instantânea de avaliações via celular ou scanner; integração ao sistema de gestão acadêmica de turmas; análise estatística detalhada das avaliações, com feedback para o professor, o aluno e a coordenação dos cursos.
\end{abstract}

Palavras-chave: avaliação formativa, tecnologia educacional, questões individualizadas.

\section{MULTIPROVA: IMPROVING ASSESSMENT WITH TECHNOLOGY}

Abstract. Efforts to evaluate students' learning processes, offering individual feedback demand an efficient strategy to deal with the large amount of output information. In this paper, we discuss a method of formative assessment based on the main features of Multiprova software: assignement of different questions for each student; use of figures, charts and equations; instantaneous grading of assessments using either mobile app or scanner; integration with SIGAA, a web based academic administration software; detailed statistical analysis of assessments, and feedback for teachers, students and academic managers.

Keywords: formative assessment, educational technology, individualized questions.

\section{Introdução}

É comum, no contexto da educação superior no Brasil, encontrarmos turmas constituídas por estudantes sem autonomia e com as mais variadas deficiências trazidas do Ensino Médio. Nas disciplinas básicas dos cursos de engenharia há ainda a problemática das turmas grandes e dos desafios conceituais intrínsecos ao aprendizado de temas como o cálculo diferencial e integral (Malta, 2004).

$\mathrm{O}$ processo de ensino-aprendizagem tem como protagonista o estudante. $\mathrm{O}$ professor é, quando muito, um facilitador e orientador do processo (Abreu, 1995). Em seu papel de orientador, uma das ferramentas de que o professor dispõe são as avaliações, entendidas como qualquer ação que busque experimentar o estado do processo de aprendizagem do estudante. Conforme o momento em que é aplicada, a avaliação pode ter caráter diagnóstico (início), formativo (meio) ou somativo (final). Para a maioria dos docentes, avaliação é sinônimo de prova aplicada ao término de um bloco de conhecimentos para inferir se o estudante atingiu determinados objetivos (Hattie e Timperley, 2007), ou seja, tem caráter somativo. Porém, a avaliação pode trazer muito mais benefícios se for feita durante o processo, permitindo correções de rumo no tempo propício (ver, por exemplo, Bransford et al (2000); Cruz et al (2011)). 
Como acompanhar o processo de ensino-aprendizagem de uma turma sem comprometer boa parte do tempo do professor na gestão das informações decorrentes dessa ação avaliativa? Neste artigo, apresentamos uma estratégia que faz uso do Multiprova, uma aplicação WEB para elaboração e gerenciamento de avaliações. O software foi criado na Escola de Ciências e Tecnologia (ECT) da Universidade Federal do Rio Grande do Norte (UFRN) para simplificar e automatizar as etapas operacionais de organização, correção, análise e compartilhamento de informações das avaliações em uma realidade de turmas grandes (mais de cem estudantes). Ele permite implementar variações na elaboração das questões, de forma que cada estudante receba questões diferentes, porém equivalentes. Em fase de testes, cerca de 15 mil provas individuais foram aplicadas nos últimos dois anos na UFRN por cerca de 20 professores em suas disciplinas. Atualmente, o Multiprova está disponível, em fase de divulgação, a todos os mais de 2200 docentes dos vários campi da UFRN através do endereço multiprova.ufrn.br. Neste texto apresentamos pela primeira vez à comunidade de usuários e à comunidade científica um artigo sobre o software e suas funcionalidades.

Como saber se os objetivos de uma avaliação foram atingidos? Quais os objetivos de uma avaliação? Uma avaliação pode ser aprimorada? Questionamentos como esses deveriam ser feitos pelos docentes em sua prática didática (ver Medeiros (1989) e Gil ( 2011)). A avaliação da avaliação é necessária, mas quase nunca é feita. A comparação entre avaliações é uma preocupação, por exemplo, dos responsáveis pelo Exame Nacional do Ensino Médio, o ENEM. Um dos principais objetivos é garantir certa equivalência entre diferentes edições do exame, uma vez que elas servirão de parâmetro para a seleção de vagas do Ensino Superior. No contexto de uma sala de aula, a comparação entre avaliações pode revelar vícios e insucessos da ação docente, permitindo que o professor a aprimore com o passar do tempo. A comparação permite também a visualização dos efeitos de eventuais mudanças metodológicas de uma turma para outra. Conhecendo melhor suas avaliações, o professor pode entregar a estudantes e gestores informações mais precisas sobre a evolução temporal de indicadores da qualidade do ensino. O impacto das avaliações no processo de ensino-aprendizagem vai depender decisivamente da qualidade das informações que são retornadas aos atores envolvidos, da celeridade dessa resposta e da gestão eficiente desse fluxo de informações (Hattie e Timperley, 2007).

Os aspectos operacionais envolvidos na avaliação podem ficar a cargo de ferramentas tecnológicas, como o Multiprova. Cabem, porém, ao professor as partes mais críticas, que são: (i) a elaboração de questões claras, tecnicamente bem estruturadas, e que suscitem o entendimento; (ii) a identificação e a formatação das informações relevantes a serem compartilhadas no feedback de cada questão. O feedback proporcionado pela avaliação serve como subsídio ao estudante, permitindo que ele otimize seus estudos, e ao professor, para que ele otimize sua ação docente, não apenas identificando os focos das suas próximas intervenções, mas também aprimorando seu instrumento avaliativo (Pereira et al, 2016). Quando não permitimos que esse ciclo se estabeleça plenamente, não teremos experimentado o inteiro potencial da ação avaliativa.

Nas próximas seções, vamos discutir o potencial de uso do Multiprova. A análise que faremos é baseada em nossa experiência de cinco anos de desenvolvimento e uso da ferramenta em disciplinas de física, informática, matemática e expressão gráfica. 


\section{Metodologia: conceitos e funcionalidades}

Em sua vida acadêmica, o professor se depara com a necessidade de ter que avaliar um mesmo conhecimento em turmas diferentes. Em geral, os professores não usam sempre as mesmas perguntas, mas buscam variações nas questões, criando os mesmos questionamentos com roupagens diferentes. É conveniente distinguir em uma questão os aspectos essenciais dos aspectos acessórios. A essência da questão contempla os conceitos envolvidos, com determinada complexidade e certo nível de dificuldade. Aspectos acessórios são, como o nome sugere, os elementos complementares da questão que podem ser modificados sem que, com isso, se altere a essência da questão. Tais elementos podem ser palavras, frases, números, equações, figuras, gráficos, etc. Vamos chamar de equivalentes questões que diferem apenas nos aspectos acessórios. $\mathrm{O}$ software Multiprova permite que o professor crie, a partir de uma matriz de questão, várias questões equivalentes com variações nos números, funções, gráficos, frases, figuras, etc, isto é, nos aspectos acessórios. Essa característica distingue o Multiprova de todas as outras ferramentas de provas atualmente disponíveis.

$\mathrm{Na}$ prática, cada estudante recebe uma questão diferente, porém equivalente. Considere, por exemplo, os dois enunciados de questões da Figura 1.

Considere a placa homogênea da figura ao lado. Sabendo que $L=18 \mathrm{~cm}$, qual é o valor da coordenada $y$ do centro de massa?
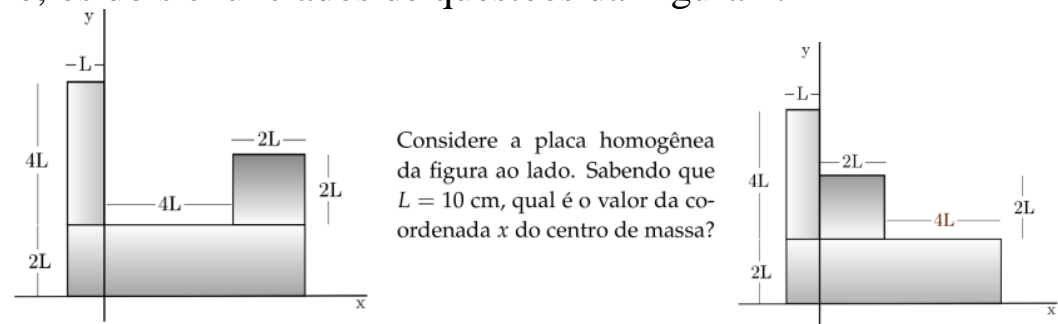

Figura 1 - Enunciados de duas questões equivalentes geradas com o Multiprova.

Perceba que o valor de L muda de uma para a outra. São diferentes também a imagem e a coordenada ( $\mathrm{x}$ ou y) pedida para o cálculo do centro de massa. Todos esses elementos citados são acessórios da questão, controlados pelo professor na tela de cadastro da questão.

Mesmo questões com enunciados totalmente diferentes podem ser equivalentes. Esse tipo de variação também pode ser implementada no Multiprova. Por exemplo, ao montar a primeira questão de uma prova, o professor indica uma lista de matrizes de questão que concorrem para ser a Questão 1. Para cada estudante, um sorteio aleatório vai definir, na lista de matrizes de questão, qual vai compor a prova como a Questão 1. Pelo fato de esse conjunto de listas de questões gerar diferentes provas, usamos, para designá-lo, o termo matriz de provas.

Ao conceber variações em uma questão, o professor apenas pressupõe que certos elementos sejam acessórios. Entretanto, tal expectativa tem que ser validada na prática pelos estudantes. É a análise do resultado da avaliação que vai definir se o aspecto é, de fato, acessório. Na seção 3, veremos um exemplo de como o Multiprova permite essa análise. É comum o professor se surpreender com a análise da avaliação, ao ver que uma das questões tidas como equivalentes foi considerada mais fácil ou mais difícil pelos estudantes. O professor termina por entender como pensam os estudantes, identificando suas dúvidas. Além disso, a partir desse feedback, o professor pode modificar suas matrizes de questões, corrigindo erros e imprecisões e fazendo ajustes nos acessórios para convergir a curva de acertos de questões equivalentes. Em análises 
como essa, passa-se a acompanhar o processo de amadurecimento das questões. Dizemos que as questões com variações perduram e isso traz vantagens.

Idealmente, uma prova feita no Multiprova teria uma variação tal nas questões que a compõem que eliminaria a necessidade da simultaneidade de sua aplicação a todos os estudantes de uma turma. Cada estudante poderia organizar seu tempo de maneira mais eficiente, evitando, por exemplo, a realização de mais de uma avaliação em um mesmo dia. Para um estudante tirar proveito das informações repassadas por um colega, ele teria que dominar as variações existentes nas questões, terminando por se envolver nos conhecimentos da questão.

Muito em breve, as avaliações do Multiprova poderão ser feitas na tela do computador ou tablet. A ideia é que a instituição destine uma sala monitorada com dispositivos executando o software de provas. O professor abriria uma janela de tempo para que os estudantes pudessem fazer a avaliação. Essa versão online do Multiprova vai permitir também que as questões sejam dinamicamente geradas. Por exemplo, o nível de uma questão poderá depender do acerto ou erro do estudante na questão anterior.

A disponibilidade permanente das questões e provas permite seu uso repetido ao longo do tempo, gerando um histórico de estatísticas que são acompanhadas pelo Multiprova. A Figura 2 traz o gráfico do histórico de acertos da uma matriz de questão da disciplina de Computação Numérica em diferentes turmas da ECT/UFRN, de diferentes semestres. Gráficos como esse permitem a comparação entre turmas, evidenciando o efeito de mudanças metodológicas na apresentação dos assuntos relativos àquela questão.

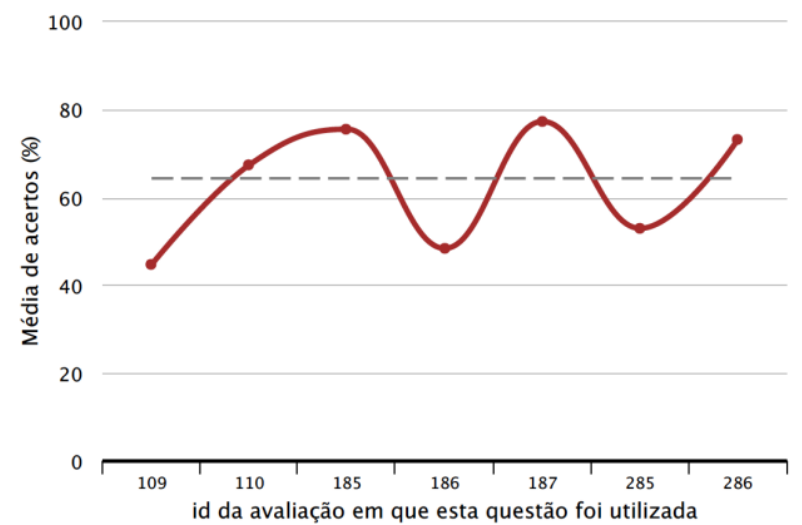

Figura 2 - Gráfico com a média de acertos de uma questão em diferentes avaliações.

\section{Discussão de uso e resultados}

\subsection{A preparação das questões}

Apresentaremos brevemente os recursos disponíveis pela versão atual do Multiprova no momento do cadastro de matrizes de questões. Os exemplos considerados são voltados para o público de ciências exatas.

O professor dispõe de um campo para o enunciado e uma quantidade arbitrária de campos de alternativa, como mostra a Figura 3. Enunciado e alternativas são utilizados para compor o corpo principal de um documento que será compilado com o editor LaTeX (página oficial: www.latex-project.org). Portanto, o usuário pode utilizar todos os recursos desse editor. A compilação é feita via webservice em um servidor onde apenas o LaTeX está instalado, por razões de segurança (Checkoway, 2010). Na tela, há 
um painel que indica os acessórios que foram definidos (variáveis, funções, listas e figuras) e que poderão ser utilizados em qualquer lugar do enunciado ou alternativas. $\mathrm{O}$ professor pode associar tags (rótulos) para organizar as questões em seu banco de dados, além de proporcionar um feedback interessante aos estudantes, conforme será discutido na próxima seção.

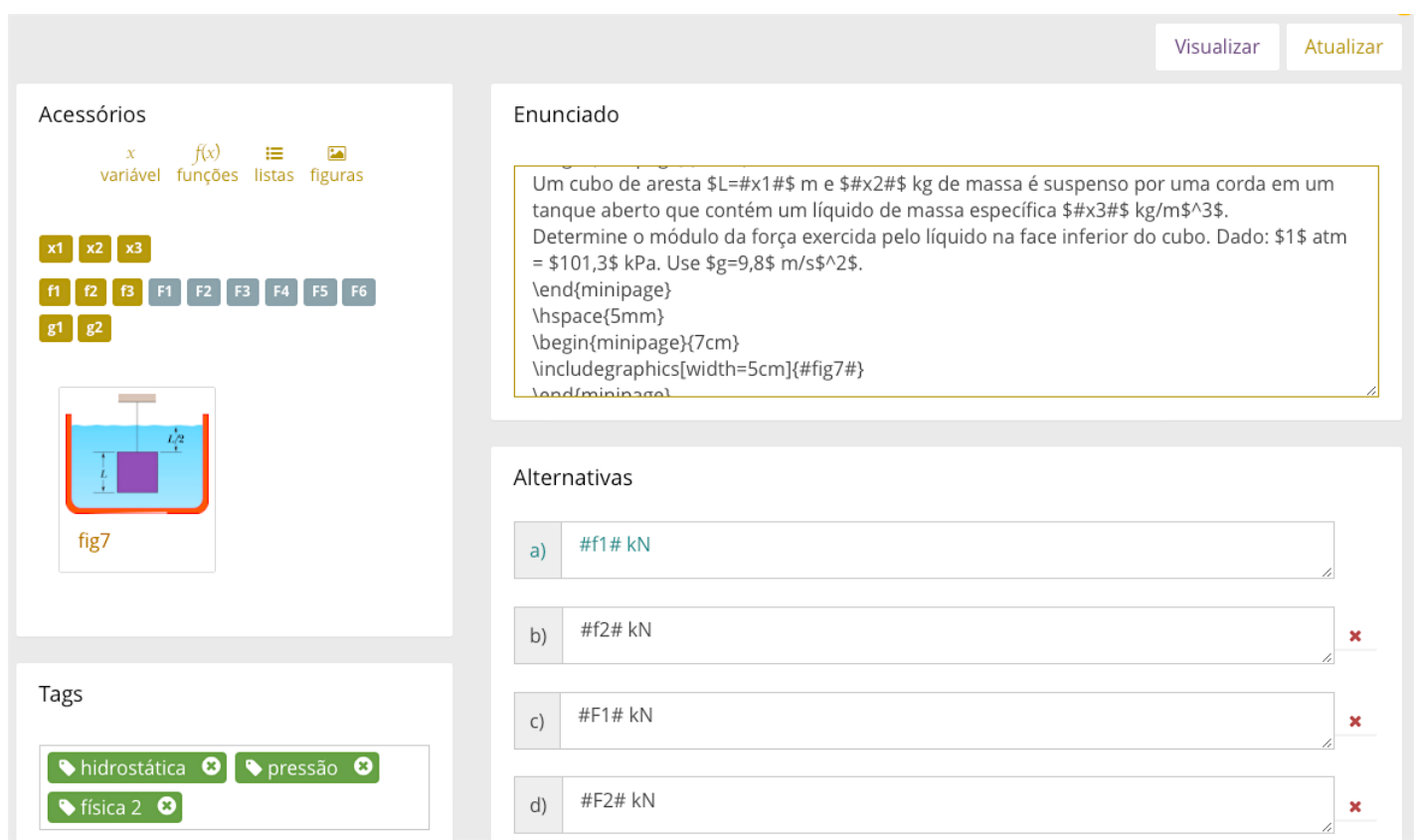

Figura 3 - Detalhe de uma parte da tela de cadastro de questões.

Nessa tela de cadastro, a alternativa de letra (a) corresponde à resposta certa. No momento da geração da prova de cada estudante as alternativas serão embaralhadas. As variáveis são denotadas por x1, x2, etc. Uma variável fica caracterizada por um valor mínimo, um valor máximo e o número de casas decimais que serão utilizadas para expressá-la. Para usar as variáveis x1, x2, etc no enunciado ou alternativas, utilizam-se os símbolos \#x1\#, \#x2\#, etc, respectivamente.

As funções são agrupadas em famílias: família $\mathrm{f}$, família $\mathrm{g}$, família $\mathrm{h}$, etc. Uma família pode conter funções calculadas em termos das variáveis (exemplo: f1 = $\left.\cos \left(3^{*} \mathrm{x} 1\right)^{*} \exp (-\mathrm{x} 2)\right)$ ou funções sorteadas aleatoriamente. O Multiprova garante que as funções dentro de uma mesma família têm valores diferentes, isto é, se dois valores coincidirem, novas variáveis são sorteadas (até um certo número de tentativas). Fizemos a modelagem em famílias de funções para contemplar uma estrutura padrão de questão (template) onde há variáveis no enunciado e se pede que se obtenha certa quantidade (resposta certa). Nas alternativas, deve aparecer a quantidade pedida (função das variáveis) e valores errados. Dentre os valores errados pode haver "pegadinhas" (que também são funções das variáveis) ou valores aleatórios (obtidos de forma inteligente e automática, tomando-se por base o valor da resposta certa e das pegadinhas). Finalmente, essas quantidades devem ser todas diferentes. Tal como no caso das variáveis, usamos os marcadores \# para inserir o valor da função no texto do enunciado ou alternativas.

Outro recurso disponível são as listas. Cada lista é um conjunto de elementos (strings) que poderão ser utilizados em qualquer lugar da questão. Por exemplo, considere três listas que trazem o símbolo de uma molécula e sua respectiva massa: $\left(\mathrm{CO}_{2}, 44\right),\left(\mathrm{NO}_{2}, 46\right),\left(\mathrm{SO}_{2}, 64\right)$. $\mathrm{O}$ n-ésimo elemento de uma lista é denotado por \#lista:n\#. Ao gerar uma prova, o Multiprova sortearia uma dessas três listas para 
compor a questão. Assim, \#lista:1\# pode vir a ser $\mathrm{CO}_{2}, \mathrm{NO}_{2}$ ou $\mathrm{SO}_{2}$, conforme a lista sorteada. Um possível enunciado seria: "Qual é a massa molecular do composto \#lista:1\#?" A alternativa correta seria: \#lista:2\#. Os elementos das listas podem ser usados na definição das funções. Isso amplia bastante as possibilidades. Por exemplo, poderíamos ter perguntas do tipo: "Quantos mols há em $10 \mathrm{~kg}$ de \#lista:1\#?". Nesse caso, a resposta seria dada em função de \#lista:2\# (massa molecular). Trocando-se a quantidade $10 \mathrm{~kg}$ por \#x1\# kg já teríamos uma variação significativa para a geração de questões equivalentes.

Para inserir figuras, o usuário deve fazer upload da imagem e associá-la à questão clicando no ícone "figuras" na tela de cadastro de questão. Após isso, ela estará disponível para compor enunciado ou alternativas, bastando que se arraste a figura para o local desejado. Ao fazer isso, aparece a instrução LaTeX para a inclusão da figura, permitindo que suas dimensões sejam ajustadas. O sorteio de figuras pode ser implementado usando as referências das figuras dentro de "listas".

Além disso, usando pacotes específicos do LaTeX (como o pgfplots), gráficos podem ser gerados dinamicamente em termos das variáveis e funções definidas na questão, resultando em gráficos individualizados!

Uma vez preparadas as questões, o professor pode montar avaliações dentro de suas turmas. A busca pelas questões é facilitada pelo uso de tags. O professor tem acesso visual rápido ao enunciado da questão, a suas tags, id e à taxa de acerto da questão até aquele momento, como mostra a Figura 4. Ao se clicar em uma questão, os detalhes de edição e do seu histórico (como na Figura 2) são apresentados.

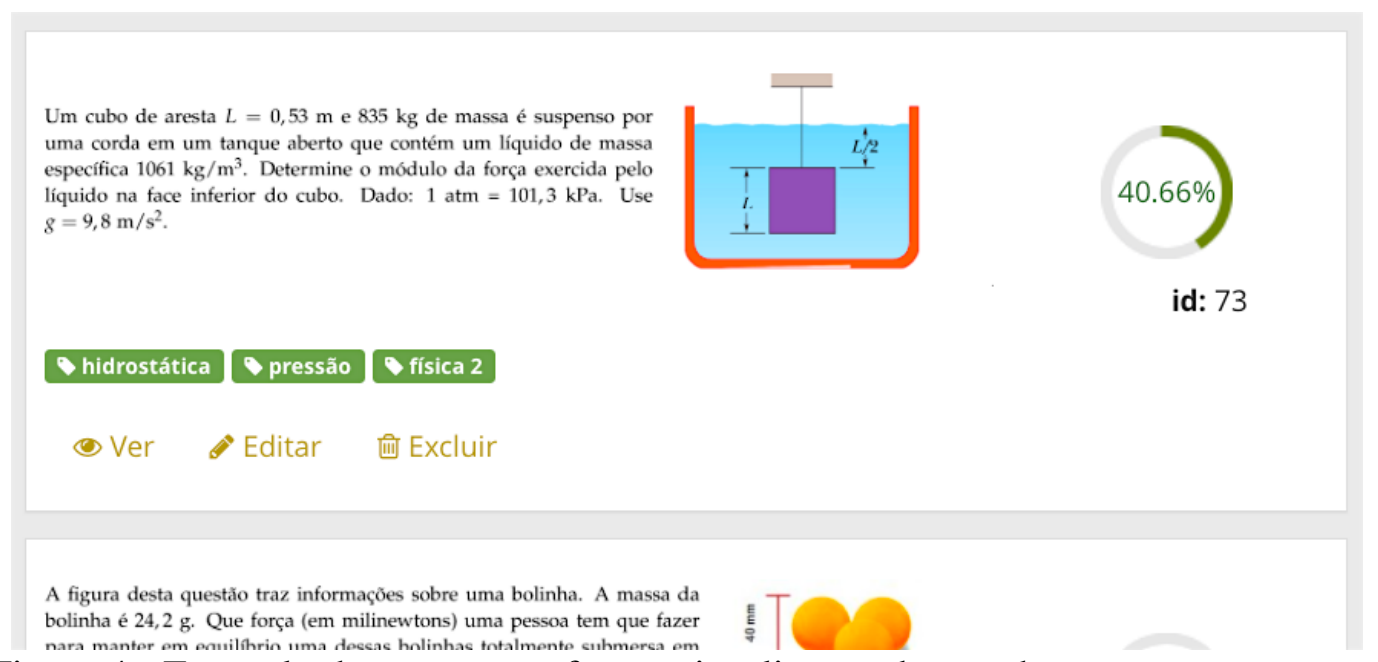

Figura 4 - Exemplo de como o professor visualiza seu banco de questões, com acesso fácil ao enunciado, tags, id e porcentagem de acerto no histórico de cada questão.

Após selecionar as questões e criar a avaliação associada a uma de suas turmas, o Multiprova se encarrega de gerar uma prova para cada estudante, que pode compor um arquivo em pdf para impressão ou pode ser aplicada individualmente em um terminal de computador. As avaliações objetivas em papel geradas pelo Multiprova podem ser corrigidas rapidamente com um celular ou scanner, utilizando soluções desenvolvidas na ECT/UFRN. Na correção por celular, o professor instala um aplicativo que corrige, com um clique, cada prova a partir de uma foto do cartão-resposta. A correção por scanner tem a vantagem de ser mais rápida: tipicamente, um scanner com alimentação automática lê mais de 30 cartões-resposta em formato A5 por minuto. A informação lida pelo celular ou scanner fica automaticamente disponível no Multiprova, bem como 
todas as análises oferecidas pelo software. Portanto, minutos após o término da avaliação, as informações terão sido compartilhadas aos professores e estudantes para que possam fazer bom proveito delas na melhoria do aprendizado.

\section{2 - Potencial didático e resultados de experiências}

Após a correção de cada avaliação, estão prontamente disponíveis ao professor: tabela com a identificação do aluno, com as respectivas respostas e o respectivo gabarito e nota; média e desvio padrão da turma na avaliação; gráfico com histograma de notas da turma e gaussiana de ajuste; gráfico de acerto por questão da turma na avaliação. Além disso, o Multiprova faz uma análise separada de cada questão, como na Figura 5. Digamos que, na elaboração da questão, além da resposta certa, na letra (a), haja uma pegadinha na letra (b), sendo as opções seguintes aleatoriamente geradas. O Multiprova gera automaticamente um gráfico da distribuição das letras marcadas pelos estudantes. Nesse gráfico, o professor pode ver quantos estudantes caíram na pegadinha, quantos chutaram, podendo alertar rapidamente a turma acerca das dificuldades identificadas. A Figura 5 exibe também o desempenho comparativo de duas questões consideradas, $a$ priori, equivalentes: alguns estudantes receberam aleatoriamente a de cor marrom, outros a de cor cinza. Por esse gráfico, ele pode se certificar se os níveis de dificuldade das questões são, de fato, os mesmos.

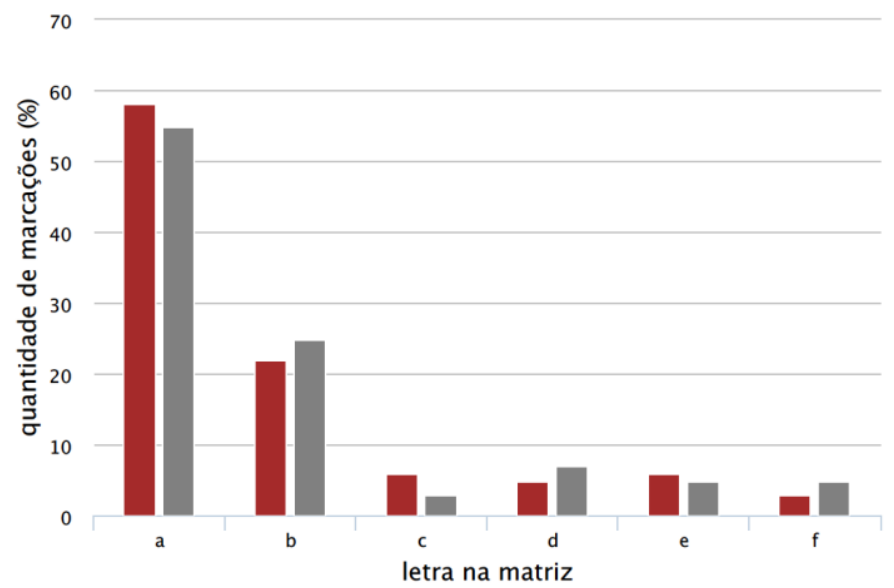

Figura 5 - Gráfico da distribuição de marcações por item para duas questões equivalentes (cor marrom e cor cinza). Destacam-se a resposta certa (letra (a) na matriz) e a pegadinha (letra (b)).

Uma associação criteriosa de rótulos às questões é um trabalho inicial que permitirá o acompanhamento transversal automático do desempenho individual dos estudantes ao longo de sua formação. Por exemplo, se os professores combinam (orientados pela coordenação do curso) de rotular as questões que lidam com vetores associando a elas o rótulo "vetores", é possível informar ao estudante a taxa de acerto acumulado nas questões envolvendo vetores nas diferentes disciplinas por ele cursadas. Será possível identificar, então, até que ponto o mau desempenho dos estudantes numa disciplina é devido a essa ou outra deficiência. Para as coordenações, será possível acompanhar o grau de domínio de certas habilidades e competências previstas nos projetos pedagógicos dos cursos. 
A partir do esforço inicial dos professores na montagem do seu banco de questões, o Multiprova produz relatórios individualizados aos estudantes, oferecendo feedback sobre cada avaliação, gráficos do histórico de avaliações, análise do desempenho transversal nos diversos aspectos marcados com rótulos, etc. A cada dia, novas estatísticas são propostas. Para as provas feitas em computador, por exemplo, vamos acompanhar o tempo gasto pelos estudantes em cada questão de uma avaliação.

Outro aspecto simplificador do Multiprova está em sua integração com o sistema de gestão acadêmica SIGAA (2017) utilizado na UFRN e em dezenas de universidades federais. Recentemente, esse sistema passou a disponibilizar as informações acadêmicas de forma organizada para alimentar aplicações externas. Com essa integração, descrita em Aquino et al (2014), o professor usa o login do SIGAA para entrar no Multiprova, onde passa a ter acesso dinâmico a suas turmas. Isso simplifica enormemente a emissão e divulgação de relatórios de notas. No futuro, o SIGAA permitirá também a importação de notas oriundas do Multiprova.

Neste artigo, estamos abordando o uso do Multiprova dentro de uma estratégia de avaliação continuada, onde o processo de ensino-aprendizagem dos estudantes é acompanhado através de vários testes periódicos. Nossa experiência tem mostrado os benefícios desta prática. Porém, o Multiprova é uma ferramenta e pode atender a diversas metodologias. Uma possibilidade interessante para instituições é disponibilizar avaliações às quais os estudantes poderiam se submeter a qualquer tempo para terem uma ideia do seu nível de domínio de diferentes assuntos. Por exemplo, uma avaliação com algumas questões análogas à mostrada na Figura 1 pode atestar o domínio do cálculo de centro de massa de figuras simples. Numa variação desse uso, avaliações poderiam ser criadas para determinar se um estudante pode, ou não, ser dispensado de cursar uma dada disciplina ou curso de nivelamento. Na ECT/UFRN, o Multiprova passou a ser utilizado no início de cada semestre para mapear as deficiências dos cerca de seiscentos alunos ingressantes no Bacharelado em Ciências e Tecnologia (BCT) da ECT/UFRN.

Diferentes estratégias foram experimentadas pelos professores. Vários relatam as vantagens do uso do Multiprova em substituição às listas de exercícios, com ganhos didáticos sensíveis. Alguns, visando apenas à simplificação do processo da avaliação final, remodelam os enunciados das questões tradicionais para o formato objetivo. Esse procedimento, em geral, não produz bons resultados. Na realidade, é difícil elaborar uma boa prova objetiva (Medeiros, 1989). Dentre outros aspectos, é preciso dosar bem o tempo, evitar respostas com desenvolvimentos longos e alternativas confusas ou óbvias. As variações introduzidas pelo Multiprova demandam um cuidado ainda maior, visto que uma única questão se desdobra em várias outras, todas devendo ser consistentes, de igual dificuldade, etc. Como vimos, esse é um esforço intelectual que valoriza a ação do professor e lhe traz frutos, diferentemente do esforço mecânico de corrigir provas tradicionais em busca de pedaços de raciocínios corretos.

Vários professores mantêm a proposta de avaliar apenas ao final da unidade de conhecimento, porém adotando avaliações mistas (parte objetiva e parte discursiva). Alguns aplicam um teste objetivo na aula que antecede a prova discursiva, reduzindo o peso desta última. Em ambos os casos, o uso do Multiprova traz vantagens operacionais, o que é essencial para turmas grandes dos cursos básicos, porém produz pouca influência no aprendizado, uma vez que não há tempo para a intervenção do professor.

O banco de questões individualizadas do Multiprova pode servir também a outras ferramentas para dar suporte a metodologias específicas. Por exemplo, a aplicação 
MiniTeste foi criada com o intuito de implementar a abordagem de instrução por pares (ver Mazur (1997); Mazur e Araújo (2013)) no contexto do ensino nas escolas e universidade brasileiras. Toda a parte de elaboração de questões fica a cargo do Multiprova e o banco de questões é acessado pelo MiniTeste por meio de webservice.

\section{Conclusões}

As ações avaliativas estão entre os principais instrumentos do professor para a melhoria do processo de ensino-aprendizagem dos estudantes. Dois fatores contribuem para reduzir o impacto dessas ações: falhas no compartilhamento das informações geradas; ausência de análise e acompanhamento do histórico de avaliações. Como toda construção intelectual, uma avaliação precisa ser revista e aprimorada.

O fluxo de informações resultante dessas iniciativas deve ter uma gestão eficiente, automatizada. Isso pode ser implementado com o auxílio de ferramentas tecnológicas, como o Multiprova. O uso de avaliações objetivas preparadas e gerenciadas no Multiprova é um modo eficiente de se implementar um processo de avaliação continuada. O software pode ser utilizado também em processos de seleção, certificação, dispensa de disciplinas, etc, além de atender à implementação de outras metodologias, como aquelas baseadas na instrução por pares. Um banco de questões variado, disponível a qualquer tempo aos estudantes, pode estimular o desejado envolvimento dos discentes, respeitando seu ritmo individual, sem perder de vista o seu acompanhamento por parte do professor. Em particular, essas funcionalidades podem contribuir para aperfeiçoar as experiências de ensino a distância.

Em todas as aplicações, cabe aos professores a principal tarefa: um esforço inicial de elaboração das questões e preparação das informações para serem compartilhadas. Prover tais informações dá trabalho, mas, diferentemente do esforço de corrigir centenas de provas, trata-se de um esforço inteligente que valoriza a ação do professor e permite um feedback qualificado a todos os envolvidos. Evidentemente, o sucesso do uso das tecnologias educacionais vai depender decisivamente da metodologia utilizada pelo professor.

\section{Referências Bibliográficas}

ABREU, M. C. O professor universitário em aula. São Paulo: M.G. Editora, 1997.

AQUINO, G. S. A.; SILVA, D. R. C.; BESSA, A; MARTINS, A. M., Integração da ferramenta Multiprova ao sistema integrado de gestão de atividades acadêmicas. RENOTE - Revista Novas Tecnologias da Educação, 8 (3) , 2014.

BRANSFORD, J. R., BROWN, A. L., COCKING, R. R., How People Learn (National Academy Press, Washington, 2000.

CHECKOWAY, S.; SHACHAM H.; RESCORLA, E.. Are Text-Only Data Formats Safe? Or, Use This LaTeX Class File to Pwn Your Computer. Proceedings of LEET 2010 - USENIX, 2010. 
CRUZ, E.; DIAS, H.; KORTEMEYER, G. Efeito da avaliação formativa em cursos de física em universidades brasileiras, Revista Brasileira de Ensino de Física, 33 (4), 2011.

GIL, A. C. Didática do Ensino Superior. São Paulo: Editora Atlas, 2011.

HATTIE, J.; TIMPERLEY, H., The power of feedback, Review of Educational Research, 77, 2007.

MALTA, I. Linguagem, leitura e matemática. In: Cury, H. N. Disciplinas matemáticas em cursos superiores: reflexões, relatos, propostas. Porto Alegre: EDIPUCRS, 2004.

MAZUR, E. Peer Instruction. Upper Saddle River: Prentice Hall, 1997.

MAZUR, E., ARAÚJO, I., Instrução pelos colegas e ensino sob medida: uma proposta para o engajamento dos alunos no processo de ensino-aprendizagem de Física, Cad. Bras. Ens. Fís., v. 30, n. 2: p. 362-384, 2013.

MEDEIROS, E. B. (1989). Provas objetivas, discursivas, orais e práticas. Rio de Janeiro: Fundação Getúlio Vargas, Rio de Janeiro, 1989.

PEREIRA, D.; FLORES, M. A.; SIMÃO, A. M. V.; BARROS, A., Effectiveness and relevance of feedback in Higher Education: a study of undergraduate students. Studies in Educational Evaluation, 49, 2016.

SIGAA, Sistema Integrado de Gestão de Atividades Acadêmicas. Acesso através de <www.sigaa.ufrn.br>. 